\title{
Diagnosis of chronic hepatitis E
}

Yu Linghua and Yin Xinguang

We found it interesting to read the Review by Rakesh Aggarwal (Diagnosis of hepatitis E. Nat. Rev. Gastroenterol. Hepatol. doi:10.1038/nrgastro.2012.187), ${ }^{1}$ which provided valuable clues to our current research on the diagnosis of chronic hepatitis $\mathrm{E}$.

Hepatitis E virus (HEV) is a common cause of acute viral hepatitis in developing countries, where the sanitation conditions are usually substandard. Most cases are self-limited, but some instances of chronic HEV infection occur among people who are immunodeficient, such as patients with HIV infection and recipients of an organ transplant. ${ }^{2}$ Progression of acute HEV infection to chronic hepatitis $\mathrm{E}$ in immunocompetent patients is rare, with only one case reported to date. ${ }^{3}$

With the improvement of public health facilities, we only have sporadic cases of hepatitis $\mathrm{E}$ in our area. However, we have observed several cases of unexplained long-term mild increases in serum levels of alanine aminotransferase and jaundice among patients who are immunocompetent. We speculate that some of these cases might be attributable to chronic HEV infection, but lack the confirmatory evidence. In addition, we wonder whether people who have close contact with patients who have hepatitis E can become healthy HEV carriers. To investigate these questions, we are looking for a good diagnostic assay to detect HEV RNA in the serum or stool of these patients, but most of the tests are still experimental. The Review by Aggarwal suggests that the loop-mediated isothermal amplification (LAMP) assay might be useful in this situation, as it has high sensitivity and specificity. Are there any other assays that meet these requirements, other than LAMP?

The First Affiliated Hospital of Jiaxing Medical College, 1882 Central-South Road, Jiaxing, 314001 Zhejiang Province, China (Y. Linghua, Y. Xinguang).

Correspondence to: $Y$. Linghua Yu.lh70s@gmail.com

doi:10.1038/nrgastro.2012.187-c1

Competing interests

The authors declare no competing interests.

1. Aggarwal, R. Diagnosis of hepatitis E. Nat. Rev. Gastroenterol. Hepatol. http://dx.doi.org/ 10.1038/nrgastro.2012.187.

2. Kamar, N. et al. Hepatitis E virus and chronic hepatitis in organ-transplant recipients. N. Engl. J. Med. 358, 811-817 (2008).

3. González Tallón, A. I. et al. Chronic hepatitis E in an immunocompetent patient. Gastroenterol. Hepatol. 34, 398-400 (2011). 